\title{
LA EXPRESIÓN DE LA EVIDENCIALIDAD EN LATÍN Y ESPAÑOL: VIDEOR Y PARECER
}

\section{Cadina Palachi}

UNL

Resumen: Este trabajo presenta un análisis del verbo latino videor (parecer) y del español parecer y describe las características sintácticas de las estructuras en las que se insertan. Se observa que ambos verbos poseen rasgos de evidencialidad, de manera que pueden ser considerados elementos funcionales. Describimos el evento de conocimiento y la forma en que se presenta sintácticamente la expresión de la inferencia. Las preguntas por la forma en que se realiza en las lenguas la expresión de contenidos orientados hacia el hablante constituyen un campo en expansión en la actualidad, en la medida en que comprometen la estructura funcional de la oración y forman parte de la investigación referida a la llamada periferia izquierda que provee de instrucciones a la interfaz conceptual.

Palabras clave: EvidencialidadPeriferia izquierda-Conocimiento

\begin{abstract}
This paper presents an analysis of the Latin verb videor (seem) and Spanish parecer (seem) and describes the syntactic characteristics of the structures in which they are inserted. Our work notices that both verbs have evidentiality features so that they can be considered functional elements. We describe the event of knowledge and how the expression of inference is syntactically done. Questions by the way in which languages express the speaker oriented contents constitute an expanding field because they are referred to the functional structure of the sentence and they form part of the research about the left periphery which provides instructions to the conceptual interface.
\end{abstract}

Key words: Evidentiality-Left Periphery-Knowledge 


\section{Introducción}

La forma pasiva del verbo latino video (ver) ha desarrollado características propias que lo alejan de su contraparte activa. Videor (parecer) no ha recibido la suficiente atención en la bibliografía especializada en comparación con verbos de similar comportamiento del español (parecer), del inglés (seem), del italiano (parere, sembrare) o del francés (sembler). Sin embargo, resulta interesante porque presenta características que lo acercan a los verbos copulativos y a los (semi)auxiliares; en cualquiera de sus dos valores se trata de un verbo que no selecciona su sujeto.

En el marco de la Gramática Generativa, el verbo seem y sus semejantes en otras lenguas, tal como es el caso de parecer, ha sido considerado un ítem léxico que selecciona un solo argumento en función de complemento proposicional y que es incapaz de seleccionar semánticamente su sujeto, razón por la cual, cuando el complemento es una oración con verbo conjugado, el sujeto permanece dentro de los límites estructurales de la misma, en tanto que si la cláusula seleccionada es de infinitivo, que carece de sintagma de tiempo que pueda chequear el caso nominativo del sujeto, éste se eleva al especificador del sintagma de tiempo de videor que le otorga caso pero no lo selecciona semánticamente. Por este motivo, se ha considerado a parecer como un verbo de "elevación de sujeto".

Por otra parte, dentro de otro marco teórico, la propuesta de Cornillie (2007) considera que parecer es un semi-auxiliar evidencial y que presenta la particularidad de la "transparencia", por lo que el sujeto del infinitivo también lo es del verbo conjugado parecer. Entendemos que, por caminos diferentes, tanto dentro de la Gramática de tipo Funcionalista como Generativista se ha llegado a describir a 
este verbo de una manera muy similar en lo que respecta a su comportamiento sintáctico como verbo de elevación de sujeto o bien como verbo transparente.

Además de su comportamiento sintáctico, creemos que es importante observar el significado evidencial que portan videor y parecer, por cuanto se trata de verbos que se asemejan en lo referido a su capacidad para aportar rasgos relacionados con la forma en que el hablante presenta la inferencia de conocimiento $y$ en tal sentido, tiene implicaciones para lo que Cinque y Rizzi (2008) han denominado la cartografía de las estructuras sintácticas que tiene por objetivo "to draw maps as precise and detailed as possible of syntactic configurations" y, dentro de esta línea de investigación, interesa entender la forma en que la sintaxis es capaz de codificar elementos referidos a la evaluación que el hablante hace de su proposición, problema referido a la cartografía de la periferia izquierda de la oración. La hipótesis es que hay una jerarquía universal de proyecciones funcionales que dominan los sintagmas proyectados por categorías léxicas y por encima de la oración, es importante notar que la posición de los especificadores de estos sintagmas es también universal.

El latín, que presenta cierto grado de orden libre de palabras, se plantea como un campo fecundo e interesante para investigar estas cuestiones. 


\section{Descripción de la sintaxis}

A diferencia de lo que sucede con el verbo español parecer, para el que el Diccionario de la RAE destina una entrada léxica independiente, con una acepción de percepción ("aparecer, dejarse ver") y una de opinión ("opinar, creer"), el Oxford Latin Dictionary (Glare, 1968) no presenta una entrada propia para el verbo videor. En cambio, a partir de la acepción 20 hasta la 24 de la entrada de video se consigna su valor "pasivo" e "impersonal", cuyo significado y sintaxis se alejan, como se puede observar, del rasgo semántico más relevante de percepción física, aunque conserva el de percepción intelectual, y del valor transitivo asociado con el verbo activo. Las acepciones de 21, 23 y 24 presentan el rasgo semántico de opinión.

(1) Video:

1. To perceive with the eyes, see.

20. (pass.) To appear, seem, be thought

21. (pass, with or without dat. of refl.) to seem to oneself (to), suppose or imagine (that)

23. (pass., w. neut. pron. as subj.) to seem good, right, proper, or sim.

24. (impers. pass.) to seem good, right, proper // a.w. inf.; w. acc and inf.// b.w. $u t+$ subj.

De la misma forma que en el diccionario, este verbo no ha recibido en las gramáticas del latín tanta atención como sus análogos en español, inglés o francés. Aunque es un verbo que despierta el interés de los gramáticos puesto que, como afirma Fernández Leborans (1999, pág. 2447), "se trata de un hecho excepcional; ningún otro verbo con similar estructura -los verbos psicológicos o de afección, 
por ejemplo- admite conmutación por lo de su objeto-tema (clausal o no clausal)".

Si bien parecer y también videor han sido considerados "copulativos" porque admiten una expresión predicativa que, en español, sabemos que puede ser sustituida por el pronombre neutro lo, como se observa en los ejemplos de (2) y (3):

(2) a. Ella parece inteligente

b. Ella lo parece

(3) a. sumque ornata ita ut aegra videar (Plautus, Truculentus, 475)

voy vestida así para parecer enferma

es difícil sostener que videor o parecer se comporten como otros verbos copulativos, por ejemplo, como el latino sum o como los españoles ser y estar, ya que ninguno de estos verbos admite, como sí lo hacen videor y parecer, un complemento oracional en infinitivo y el sujeto en concordancia con el verbo conjugado. De manera que supondremos que lo que distingue a videor del verbo copulativo ser/sum es su posibilidad de llevar un complemento en infinitivo con valor oracional. Efectivamente, este verbo se presenta con mayor frecuencia en contextos como los de (4), que de ninguna manera son posibles para ser o estar en español, y tampoco encontramos ejemplos en latín en la base de datos:

(4)a. Omnia subiecisse oratori uidetur Aristoteles (Quintilianus, Institutio Oratoria

2.15.16.1)

Aristóteles parece haberlo confiado todo a la competencia del orador. (Trad. Ortega Carmona)

b. non homines habitare mecum mi hic videntur, sed sues. (Plauto, Stichus, 64) 
no hombres, sino cerdos parecen habitar aquí conmigo. (me parece que habitan aquí...)

(5) a. ${ }^{*}$ Cerdos son vivir aquí./Cerdos parecen vivir aquí.

b.*Este hombre está pensar así./Este hombre parece pensar así.

Mucho menos habitual que la construcción de infinitivo resulta, en latín, la presencia de una cláusula con verbo conjugado (en subjuntivo) y nexo ut, ejemplos en (6). Aunque en español parecer admite con naturalidad oraciones con verbo conjugado en indicativo y nexo que:

(6)a. visum est ut te facerem certiorem.(Cic., Ad fam, 8.5.2.5)

Parece que te he ayudado a decidirte.

b. sed scis ita nobis esse visum ut isti ante legerent.(Cic., Ad Attic., 12.51.2.6)

pero sabes que me parece que aquellos debieran leer antes.

c. patribus id tempus reipublicae uisum est ut per ueteres et expertos bellique peritos imperatores res publica gereretur; (Liv., AUC, 27.6.10.2)

Los senadores estimaron que las circunstancias del Estado exigían confiar la dirección de los asuntos públicos a generales veteranos, experimentados y dotados de conocimientos bélicos. (Trad. Villar Vidal)

En (6) se observa que videor permite como su argumento una oración con $u t$ y verbo conjugado con su propio sujeto en caso nominativo, aunque debemos mencionar que esta estructura no resulta tan frecuente como la cláusula en infinitivo. En este sentido vemos una diferencia notable con 
el español parecer, que admite la construcción con que + verbo conjugado en indicativo + clítico dativo y en cambio, cuando lleva la construcción con infinitivo no permite la presencia del dativo (salvo un tipo de construcción que comentaremos más abajo). Aunque vamos a señalar que además, en español, el verbo parecer se utiliza con esta construcción en presente y tercera persona del singular; en latín, en cambio, los ejemplos que hemos encontrado son con visum est en pretérito y en tercera persona del singular:

(7)a. Parece que las mujeres organizaron una revolución.

a'. ?Pareció que las mujeres organizaron una revolución.

b. Las mujeres parecen haber organizado una revolución.

Descartamos entonces la posibilidad de considerar a videor como verbo copulativo por las diferencias sintácticas que presenta con sum. Por otra parte, no nos ocuparemos de la construcción de videor + oración encabezada por $u t \mathrm{y}$ verbo conjugado en modo subjuntivo, que, como hemos visto, no es muy habitual en latín y se diferencia de la construcción en español que lleva parecer en presente y la oración con verbo conjugado en indicativo. Este tipo de estructuras tampoco ha recibido suficiente atención en la bibliografía, sin embargo, aquí dejaremos la cuestión para próximos trabajos. Consideraremos la construcción de videor + cláusula de infinitivo. 


\section{Características de la construcción videor + infinitivo}

En este trabajo estamos interesados en desmontar el significado de videor como verbo evidencial capaz de insertarse en sintagmas funcionales de la periferia izquierda ligados al discurso y con efectos en la sintaxis oracional: por este motivo resulta mucho más productivo analizar la construcción con infinitivo y dativo. Tal como señala Givón (1993) citado por Cornillie (2007, pág. 12), "cuanto más orientada hacia el hablante la percepción del evento, más extensa será la integración de dos proposiciones en una sola", es decir que la construcción de verbo conjugado + infinitivo tiene más probabilidades de tener una orientación hacia el hablante que las de verbo + cláusula con que. Para observar la contribución que hace videor a la lectura de evidencialidad del evento que compromete la orientación hacia el hablante, entendemos que la presencia del dativo en estructuras con infinitivo contribuye a que prevalezca una lectura de este verbo como evidencial.

Dado que venimos estableciendo comparaciones, que se han mostrado interesantes, entre el latín y el español, vamos a considerar una vez más la forma de la construcción de videor y parecer. En (8) retomamos nuestro ejemplo (7) para experimentar qué sucede en español con la presencia del dativo:

(8)a. Me parece que las mujeres organizaron una revolución.

b. *Las mujeres me parecen haber organizado una revolución.

(8) nos permite observar que en español la presencia del dativo de opinión es posible con la estructura de parecer 
+ oración con verbo conjugado y, en cambio, no está permitido en presencia del infinitivo.

En latín este comportamiento es diferente, como se puede ver en (9):

(9)a. Mihi uidetur condicionem mutare quod his locis uerba coniungimus. (Quint, Inst Or., I.5.27.1)

Me parece que cambia la condición, porque en estos lugares hacemos uniones de palabras. (Trad. Ortega Carmona) (*me parece cambiar la condición)

b. Quare mihi non inuenuste dici uidetur aliud esse Latine, aliud grammatice loqui. (Quint, Inst. Or., I.6.27.3)

Por lo cual me parece que se dice, no sin cierta gracia, que una cosa es hablar latina y otra gramaticalmente. (Trad. Ortega Carmona) (*me parece decirse)

c. illa, Caecili, contemnendane tibi videntur esse,...? (Cic, In Caecil., 35.7)

¿Estos asuntos te parece, Cecilio, que deben ser consideradas? (*ite parecen deber ser considerados?)

Las traducciones al español de los ejemplos presentados en (9) recurren a estructuras con nexo subordinante que + verbo conjugado (en indicativo) para poder mantener la presencia del dativo de opinión.

Entendemos, entonces, que existe una diferencia de comportamiento referida particularmente a una de las acepciones posibles de videor. Tal como señala Fernández Leborans, resulta relevante en relación con la sintaxis considerar la diferenciación semántica entre el uso de "parecer como verbo de "percepción" de su uso como verbo de "cognición" u "opinión" (Fernández Leborans, 1999, pág. 2443). Teniendo en cuenta que "el verbo de "cog- 
nición" u "opinión" requiere la presencia del argumento experimentante", vemos que videor + dativo puede ser interpretado como verbo de opinión/cognición y no como verbo de percepción.

La primera diferencia sintáctica con el español es que parecer, como hemos visto, no permite la realización del dativo cuando el complemento se encuentra en infinitivo y, en consecuencia, el valor semántico de opinión. Se debe mencionar aquí una estructura como la siguiente, que sin embargo es diferente de las posibles en latín:

(10) Me pareció oír una explosión en la calle.

En (10) la presencia del clítico dativo en el contexto de un verbo de percepción física como oír es perfectamente aceptable y parecer no adquiere el valor de un verbo de opinión, sino que prevalece el significado de percepción física de oír y el dativo carece del rasgo relacionado con la opinión. Es preciso notar además que la misma construcción admite otros verbos de percepción como ver, escuchar. En esta estructura, parecer va siempre en tercera persona del singular y no hace la concordancia con el sujeto del infinitivo, como sí es posible cuando el sujeto del infinitivo lo es también del verbo conjugado. Además es notable que el clítico dativo debe necesariamente coincidir con (estar controlado por) el sujeto del infinitivo (11c y d):

(11)a. *Nos parecieron oír su voz y una explosión.

b. ${ }^{*}$ Me pareciste decir la verdad.

c. ¿'Te pareció oír un ruido en la calle?

d. *Te parecí oír un ruido en la calle. 
En latín, videor se comporta como un verbo de opinión en presencia de infinitivos y admite el dativo. En este tipo de estructuras es capaz de llevar también el verbo en forma personal en concordancia con el sujeto, que es además el sujeto lógico del infinitivo, y puede llevar un clítico dativo que es diferente del sujeto del verbo videor y del infinitivo:

(12)a. Ergo inridere ne videare et gestire admodum; noli facere mentionem te $<$ has $>$ emisse. (Plauto, Mostellaria, 812)

Por lo tanto, para que no parezcas burlarte y hacer gestos de este modo; no quiero hacer mención de que tú las compraste.

b. an tibi irasci tum videmur, (Cicero, Tusculanae Disputationes 4.55.2)

¿Entonces te parece que estamos enojados? ( ${ }^{*}$ Entonces te parecemos estar enojados?)

c. omnes videntur scire (Plautus, Aulularia, 114) todos parecen saberlo

d. Vt, quia te tango, mel mihi videor lingere. (Plautus, Casina, 458)

Porque al tocarte me parece que estoy lamiendo miel. $\left({ }^{*}\right.$ me parezco lamer miel)

En cuanto a la flexión temporal, hemos visto que la construcción en pasado con videor (visum est + oración con verbo conjugado) es de tercera persona del singular. Sin embargo, videor en pasado también admite la construcción de infinitivo con el clítico dativo y la concordancia con el sujeto de infinitivo; como se puede observar en los ejemplos de (13), la traducción literal al español no es posible ya que, según se puede concluir de esta descripción, 
en español la construcción de parecer + dativo + infinitivo se encuentra mucho más restringida que en latín.

(13)a. Phanostrata. Audire vocem visa sum ante aedis modo mei Lampadisci servi. (Plauto, Cistellaria, 543)

Me parece haber oído aquí a la puerta la voz de mi esclavo Lampadión. (Trad. González-Habba)

(*Parecí haber oído la voz...)

b. mercari visus mihi sum formosam capram; (Plauto, Mercator, 229)

Me parecía que compraba una cabra muy hermosa (Trad. González- Habba) (*Me parecí haber comprado...)

c. verum illa nobis abhorrere ab usu oratorio visa sunt. (Cic, De Inv, 1.77.6)

en verdad, nos pareció que aquellas cosas son incompatibles con el uso de la oratoria (*aquellas cosas nos parecieron ser incompatibles...)

También encontramos estructuras con el verbo conjugado en futuro y en pretérito imperfecto y con infinitivos en diferentes voces y tiempos, por ejemplo, el infinitivo de futuro activo scituri esse:

(14)a. colloqui videbamur in Tusculano cum essem; tanta erat crebritas litterarum._(Cic, Ad Atticum, 13.18.1.2)

We seemed to be talking to one another, when I was at Tusculum, so frequent was the interchange of letters. (Trad. Winstedt)

b. sed videbamur omnia biduo triduove scituri. (Cic, Ad Atticum, 7.23.3.4)

It would seem that we shall know all in a day or so. (Trad. Windstedt) (*parecíamos haber de conocer todo en dos o tres días) 
c. et eum qui tibi inimicissimus esse videbitur (Cic, In Verrem, 2.2.152.7)

y este que te parecerá que es el mayor enemigo.

d. Quod si haec ratio rei gerendae periculosa tibi esse videbitur (Cic, Ad Fam., 1.7.6)

But if this plan of procedure in the business seems to you to be dangerous (Trad. Williams) 


\section{Evidencialidad. El evento de conocimiento:}

El uso de videor con infinitivo + dativo tiene claramente un significado relacionado con la forma en que se infiere conocimiento; en este trabajo proponemos que es un significado de evidencialidad. El concepto de "evidencialidad" resulta muy cercano al de "modalidad epistémica", pero no deberían confundirse. Señala Cornillie que:

"los hablantes a menudo comentan el contenido proposicional de manera de guiar a su interlocutor hacia una interpretación viable de lo que se dice o para atenuar la afirmación con la intención de no perjudicar su propia posición. Dado que el hablante quiere evitar el riesgo de perder contacto, a menudo sugiere que la proposición afirmada se basa en cierto modo de conocimiento y también indica cuánta certeza tiene de la verdad de su proposición. Estas dos modalidades de la comunicación tienen sus propios conceptos en la lingüística moderna: la evidencialidad se refiere a 'la encodificación (del tipo) de fundamento del hablante para la realización de su acto de habla' (Faller 2002:2), mientras que la modalidad epistémica se refiere a la 'evaluación de las oportunidades de que cierto estado de cosas hipotético en consideración (o algún aspecto de éste) va a ocurrir, está ocurriendo o ha ocurrido en un mundo posible' (Nuyts, 2001b:21)." (Cornillie, 2007, pág. 1)

Talmy (2000:2) propone encontrar "los patrones y los procesos por medio de los cuales el contenido conceptual se organiza en el lenguaje" o "cómo el lenguaje estructura el contenido conceptual". Según este autor, algunas categorías se consideran centrales porque constituyen el sostén de los sistemas conceptuales y suelen ser universales, se trata de 
aquellas relacionadas con la organización del espacio y el tiempo, el movimiento y la locación, la causalidad y las categorías atribuidas a los agentes cognoscentes.

Entonces, para comprender mejor el concepto de evidencialidad es necesario describir el evento de conocimiento o de inferencia de conocimiento que tiene, al menos, los siguientes componentes:

-sujeto cognoscente

-objeto conocido

-fuente de conocimiento

-modo de conocimiento

-evaluación sobre el conocimiento

La expresión sintáctica de este evento puede realizarse de diferentes maneras, videor es un elemento sintácticamente capaz de configurar un evento de conocimiento, de la siguiente manera: el sujeto cognoscente debe necesariamente estar expresado sintácticamente y la tendencia, según hemos visto, es mediante el clítico dativo; el objeto es la proposición en sí misma (que en latín se expresa en infinitivo) y la evaluación se manifiesta mediante elementos léxicos modales epistémicos de los que no nos ocupamos aquí. Finalmente, la fuente del conocimiento y el modo son dos aspectos íntimamente ligados entre sí que pueden no estar presentes en el enunciado, pero el verbo videor encodifica en sí mismo la noción semántica de que la fuente del conocimiento es procesada por el sujeto a partir de la evidencia indirecta o inferencia cognitiva.

Hay varias posibilidades de presentar la relación con las fuentes de conocimiento que, según Willett (1988) citado por Cornillie (2007), dependen de la forma en que se ha adquirido la información:

-de manera directa (vi que...), 
-de manera indirecta:

1) por medio de la escucha (escuché que...), o

2) inferida por el hablante a partir de la percepción intelectual (me parece que...).

Se observa que la manifestación de la evidencialidad puede hacerse por otros medios sintácticos (infiero que..., se deduce que..., etc.), aunque en este trabajo nos ocupamos sólo del valor evidencial presente en videor + dativo + infinitivo. Tal como señala Bybee (1985:184), de manera estricta, los "evidenciales pueden ser definidos en general como marcadores que indican algo acerca de la fuente de información de la proposición" Los evidenciales muestran que el hablante tiene "evidencia" o tiene un tipo de evidencia de una proposición.

Nos parece útil distinguir, como propone Cornillie (2007) en lo que respecta a las nociones expresadas por los elementos lingüísticos relacionados con la evidencialidad:

-La manifestación de la fuente indirecta de conocimiento

-El tipo de compromiso del hablante con la inferencia resultante

-La relación de intersubjetividad entre el hablante y el oyente, concebido también como un sujeto cognoscente

Analizaremos construcciones que permiten ver cómo se presenta la información que introduce videor según estos tres ejes. 


\subsection{Fuente:}

La presencia de videor + dativo indica que la fuente de la que emana el conocimiento de la situación expresada por la estructura de infinitivo no es percibida por los sentidos, sino inferida indirectamente a partir del conocimiento o intelectualmente, aunque pueda y quizás deba, haber una situación vista o conocida previamente. En (15)a. vemos que el sujeto cognoscente infiere la falta de recursos a partir de una observación hecha previamente y en b. se pregunta al interlocutor cómo hizo la inferencia en cuestión:

(15)a. ut, quotiens esset occasio, extemporales eorum dictiones his uelut emblematis exornarentur: quo quidem (neque enim eius rei iudicium differre sustineo) summam uidebantur mihi infirmitatem de se confiteri. (Quintilianus, Inst. Or., 2.4.28.2)

de modo que cuantas veces se les ofrecía la oportunidad, se adornaban como con estos ropajes las amplificaciones improvisadas: con lo cual ciertamente (y no me resigno a diferir mi juicio sobre ello) me parece que daban testimonio de la altísima pobreza de ellos mismos. (Trad. Ortega Carmona)

b. Sed scire ex te pervelim quam ob rem qui ex municipiis veniant peregrini tibi esse videantur.(Cic, Pro Sulla, 23.4)

But I should like exceedingly to know from you, how it is that those men who come from the municipal towns appear to you to be foreigners (Trad. Yonge)

\subsection{Compromiso del hablante:}

Dado que la proposición no se conoce sino que se infiere, es posible observar diferentes grados de compromiso con la evidencia. En (16a) la presencia de quidem 
(ciertamente) permite suponer un grado bastante alto de compromiso con la inferencia. En (16b), el hablante sugiere que no es lo mismo buscar consejo seguro (certi) que pedir una opinión (sed quid videatur): el sed marca claramente esta diferencia. En (16c), se niega la opinión de los sujetos cognoscentes (vobis) mostrando que una argumentación puede producir un cambio en tal inferencia:

(16)a. quamquam facienda mentio est, ut quidem mihi videtur, duorum adulescentium, qui si diutius vixissent, magnam essent eloquentiae laudem consecuti. (Cic, Brutus, 279.10)

b. itaque a te nihildum certi exquiro sed quid videatur, denique ipsam $\dot{\alpha} \pi \circ \varrho i \alpha \nu$ tuam cupio cognoscere. (Cic, Ad Atticum, 7.12.4.6)

So I don't look to you for positive advice: but only for your opinion. In fact I want to know how the dilema presents itself to you. (Trad. Windsted)

c. atque hoc haud videtur veri simile vobis, at ego id faciam esse ita ut credatis.(Plautus, Mostellaria, 93)

seguro que no os parece muy exacta la comparación, pero ya veréis cómo hago que cambiéis de opinión y consigo probar que es cierto lo que digo. (Trad. GonzálezHaba)

\subsection{Relación de intersubjetividad:}

Hemos visto en los ejemplos presentados que el sujeto cognoscente puede coincidir o no con el sujeto de la enunciación. En este sentido, la intersubjetividad se refiere a la conciencia que tiene el hablante de que el otro participante es también un sujeto hablante y, fundamentalmente, cognoscente. La evidencia puede ser compartida o no por el hablante y el oyente, generalmente el uso del 
verbo videor con un dativo de segunda persona apunta a asegurarse de que el oyente está compartiendo o no la misma evidencia y realiza las mismas inferencias cognoscitivas que el hablante:

(17)a. quid tibi ego aetatis videor? (Plautus, Mercator, 290)

¿Qué edad te parece que tengo? (lit: ¿de qué edad te parezco?)

b. in quae corpora si nullus tibi forte videtur posse animi iniectus fieri, procul avius erras. (Lucrecio, DRN, 2.739)

Y si acaso te parece que sobre tales cuerpos no se puede llevar a cabo ningún avance de la inteligencia, andas muy descarriado. (Trad. Socas) 


\section{Evidencialidad y periferia izquierda}

Es claro que videor y parecer portan un significado de evidencialidad que presenta la cláusula como un evento inferido y que el clítico dativo constituye un elemento sintáctico relevante para la interpretación de estos verbos. Sin embargo, en este trabajo hemos comprobado que existe una diferencia en el comportamiento sintáctico de videor y parecer con respecto a la transparencia. El verbo latino permite la elevación de sujeto en presencia del clítico, en tanto que el español con dativo y, por lo tanto con su valor evidencial, tiende a presentar una cláusula con nexo y verbo conjugado (sin elevación de sujeto).

La hipótesis fuerte que se sostiene en el marco del proyecto cartográfico es que el tipo y orden de sintagmas funcionales que dominan las proyecciones léxicas y la cláusula es universal y la presencia de un tipo de sintagma funcional en una lengua es suficiente para postular que tal elemento existe en todas las lenguas, aún en aquellas en que no se presente un elemento (morfema dependiente o independiente) que encarne tal significado. Cinque (2006:12) propone que

the functional portion of the clause, in all languages, is constituted by the same, richly articulated and rigidly ordered, hierarchy of functional projections, a subset of which is shown in (3):

(3) $\operatorname{MoodP}_{\text {speech act }}>\operatorname{MoodP}_{\text {evaluative }}>\operatorname{MoodP}_{\text {evidential }}$ $>$ MoodP $_{\text {epistemic }}>\mathrm{TP}($ Past $)>\mathrm{TP}($ Fut $)>$ MoodP $_{\text {irrealis }}>$ $\operatorname{ModP}_{\text {alethic }}>\operatorname{AspP}_{\text {habitual }}>\operatorname{AspP}_{\text {repetitive }}(\mathrm{I})>$ $\operatorname{Asp} P_{\text {frequentative }}(\mathrm{I})>\operatorname{ModP}_{\text {volitional }}>\operatorname{AspP}_{\text {celerative }}(\mathrm{I})>$ $\mathrm{TP}($ Anterior $)>A s p P_{\text {terminative }}>\mathrm{AspP}_{\text {continuative }}>$ AspP $\mathrm{P}_{\text {retrospective }}>\mathrm{AspP}_{\text {proximative }}>\mathrm{AspP}_{\text {durative }}>$ $\mathrm{Asp}_{\text {generic/progressive }}>\mathrm{AspP}_{\text {prospective }}>\mathrm{ModP}_{\text {obligation }}>$ 


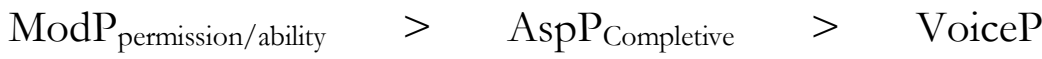
$>\operatorname{AspP}_{\text {celerative }}(\mathrm{II})>\operatorname{AspP}_{\text {repetitive }}($ II $)>\operatorname{AspP}_{\text {frequentative }}(\mathrm{II})$

Entendemos que videor y parecer se insertan por sus

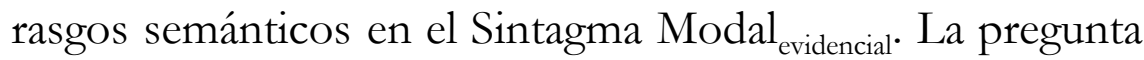
es si ambos verbos, a pesar de sus diferencias sintácticas, pueden entenderse como morfemas funcionales. Según nuestra hipótesis, videor con dativo e infinitivo es claramente un verbo modal funcional, razón por la cual forma una unidad con el infinitivo capaz de exhibir transparencia en cuanto a su sujeto. En cuanto a parecer con dativo, entendemos que podría también postularse que se trata de un morfema funcional que se inserta en el mismo sintagma que videor. Sin embargo, consideramos que esta hipótesis debe ser puesta a prueba y dejamos este aspecto para futuras investigaciones. 


\section{Conclusiones}

En este trabajo hemos propuesto un análisis de videor como verbo evidencial, cuando se presenta con una estructura de infinitivo y un dativo de opinión. La comparación con el español parecer muestra que, si bien existen similitudes en cuanto al rasgo de significado evidencial, estos verbos tienen una sintaxis diferente cuando llevan el clítico dativo. Videor admite la presencia del clítico cuando se construye con la cláusula de infinitivo, en tanto que parecer lleva el dativo en presencia de la oración con nexo (que) y verbo conjugado en modo indicativo. Si bien en latín también se registra la construcción con oración introducida por el nexo ut y verbo conjugado, éste debe ir en subjuntivo. Por otra parte, este tipo de contexto sintáctico es poco habitual.

Entendemos que los mecanismos lingüísticos, sintácticos, para la expresión de un evento de conocimiento no son estrictamente idénticos en todas las lenguas, aunque suelen intervenir los mismos componentes. Videor tiene alcance sobre la construcción de infinitivo otorgándole un significado no aseverado, sino inferido por un sujeto cognoscente que se presenta en forma de dativo. Se trata de un morfema funcional que se inserta en el Sintagma Modal $_{\text {evidencial }}$ en la periferia izquierda de la cláusula, desde donde da instrucciones a la interfaz conceptual. 


\section{Bibliografía}

Aikhenvald, Alexandra (2004) Evidentiality, Oxford, OUP

Baños Baños (Editor, 2009) Sintaxis del latín clásico, Madrid, Liceus

Bosque y Gutiérrez Rexach (2009) Fundamentos de Sintaxis Formal, Madrid, Akal

Bybee, Joan (1985) Morphology: a Study of the Relation between Meaning and Form, Amsterdam, Philadelphia, John Benjamins Publishing Company

Cinque, Guglielmo (2006) Reestructuring and Functional Heads, Oxford, OUP

Cornillie, Bert (2007) Evidentiality and Epistemic Modality in Spanish (Semi)-Auxiliaries. A Cognitive-Functional Approach, Berlin, Mouton de Gruyter

Di Tullio, Ángela (2010) Manual de gramática del español, Bs.As., Waldhuter editores

Fernández Leborans (1999) "La predicación: las oraciones copulativas" en Bosque y Demonte (Directores, 1999) Gramática Descriptiva de la Lengua Española, Madrid, Espasa

Glare, P.G.W. (Editor, 1982) Oxford Latin Dictionary, Oxford, At the Clarendon Press

Pinkster, Harm (1995) Sintaxis y semántica del latín, [Traducción de Esperanza Torrego y Jesús de la Villa, Madrid, Ediciones Clásicas]

Ridruejo (1999) "Modo y modalidad. El modo en las subordinadas sustantivas", en Bosque y Demonte (Directores, 1999) Gramática Descriptiva de la Lengua Española, Madrid, Espasa 
Talmy, Leonard (2000) Toward a Cognitive Semantics, Vol.1 Concept Structuring Systems, Cambridge, The MIT Press

Talmy, L (2000) Toward a Cognitive Semantics, Vol. 2 Typology and Process in Concept Structuring, Cambridge, The MIT Press

Torrego, Esperanza (2007) "Los esquemas de complementación de los verbos de "ver' en latín" en Torrego, Baños, Cabrillana y Méndez Dosuna (Editores, 2007) Praedicativa II: Esquemas de complementación verbal en griego antiguo y en latín. Universidad de Zaragoza. Monografías de Filología Latina 15

Torrego, Esther (1996) "Experiencers and raising verbs" en Freidin, Robert (Edit, 1996) Current issues in comparative grammar, Dordrecht, Kluwer Academic Publishers

Traducciones:

Livio, Tito Historia de Roma desde su fundación, Traducción y notas Villar Vidal, José Antonio, Madrid, Gredos, 1990

Quintiliano, Marco Fabio Instituciones Oratorias, Traducción y comentarios Ortega Carmona, Alfonso, Salamanca, Publicaciones Universidad Pontificia de Salamanca, 1996

Lucrecio, La Naturaleza, Presentación, traducción y notas de Socas, Francisco, Madrid, Gredos, 2010

Plauto, Comedias, Introducciones, traducciones y notas de González-Haba, Mercedes, Madrid, Gredos, 1992 
Cicero, Marco Tulio, Letters to Atticus, English translation by E.O. Winstedt, Oxford, The -L.O.E.B Classical Library, 1912

Cicero, Marco Tulio, The Letters to his Friends, English translation by Glynn Williams, Massachussets, The LOEB Classical Library, 1927

Cicero, Marco Tulio, Pro Sulla, English Translation by Yonge, Charles Duke, George Bells and Sons, 1856. urn:cts:latinLit:phi0474.phi015.perseus-eng1 